\title{
Low-Cost Bus Tracking System Using Area-Trace Algorithm
}

\author{
${ }^{1}$ Darshan Ingle, ${ }^{2}$ Saumya Omanakuttan \\ ${ }^{1}$ Assistant Professor, Computer Engineering, SIESGST Nerul, Mumbai University, India \\ ${ }^{2}$ Assistant Professor, Information Technology, SIESGST Nerul, Mumbai University, India
}

\begin{abstract}
This paper proposes a low-cost Bus Tracking System that uses GPS and allows the user to know the bus location on their mobile phone. It has an advantage that it does not use any internet data for the users to be notified. Instead, it uses only a simple sms in a specified format to be sent to the server. It also sends the user, the bus's expected arrival time. The system maintains a track of people travelling in the bus as well as the ticket fare from each required source to required destination. All this information is sent to the user on the receipt of a single sms to the server. This technology aims at providing a low cost solution to the problems faced by common man using the public transport thereby addressing the issues and solving them successfully. The aim is to present the work to the Indian Government which can help the struggling life become more smooth and functional.
\end{abstract}

Keywords: GPS, Area-Trace Algorithm, Haversine formula, Traffic Management System.

\section{Introduction}

Technology always aims at reducing the human efforts at a cost which turns out to be expensive. The time resource of a common man is a vital parameter for him to be successful in his work. This paper aims in developing a technology which will be available and beneficial to common man at a very low cost. It deals with developing a Bus tracking System which can locate the desired bus number, its source and destination, the maximum count of seating and standee capacity of a bus and the current count of passengers in the bus. This technology will help the common to predict the location of the bus, its expected arrival time, its travel fare and the vacancy of seats in the bus. All the above services will be made available at a single outgoing message from the mobile phone.

As per the Telecommunications statistics in India, the total number of mobile phone subscribers have reached 875.48 million as on October 2013 which is $73.32 \%$ of the entire population. When we think of a city like Mumbai, mobile phone is the basic necessity of a person. However, Mumbai being a metropolitan city, has a blend of rich class, middle class and poor class population. According to the data released by the 'Indian And Mobile Association Of India (IAMAI)' 2013 report, Mumbai ranks no 2 with 12 million internet users. However, still, not every mobile user in Mumbai has an internet connection which indirectly means that they are not having an access to the GPS.

Reports speak that almost $88 \%$ of the commuters in Mumbai use public transport of which almost $50.23 \%$ travel by Brihanmumbai Electric Supply And Transport (BEST) buses. This makes the transport of crucial importance. It is very important for the transport to function smoothly throughtout. However, there are many factors like traffic jams, heavy population, etc which make the functioning a bit lethargic. This has led as a source of motivation for us to think of some system that can be of fruitful use for the common man.

The paper aims at making a Bus Tracking System which is capable of producing a bus's location with the help of GPS and provide the same to the mobile phone users without using the mobile internet data. This aims at sending the bus location to the requesting user in a particular text format. It also sends the user the expected arrival time for the bus to arrive at the specified bus stop. Also, it maintains a count of the maximum seating and standee capacity of the bus and with the help of sensor, it also sends the number of vacant seats in the bus. This helps the user in taking decisions like whether the bus is late or on time, should the wait time for the bus be considered or some other means of transport can be considered, is there a seat vacancy in the expected arriving bus, etc. Thus the services are offered to the all the users which facilitates to save their time and effort. Also, user can get the information about the ticket fare from the source to destination.

\section{Related Work}

The various GPS tracking devices available in the market are as follows:

a. TrackPort GPS Vehicle Tracker [12]

It is a basic plug and play GPS tracking device which is available in the market. It can instantly locate your vehicle at anytime from anywhere. This GPS car tracking device sets up in seconds and allows to point vehicle location anywhere in the world. It is capable of providing real time speed and location alerts via email. It does not require any installation and can be easily moved from car to car. 
b. HCT Micro GPS Tracker [13]

It is a covert, hardwired GPS vehicle tracker which is completely invisible in any car or truck. It is a lowcost tracker and is capable of monitoring your vehicle on any computer or a mobile device. It has a lightning fast 30 seconds update rate which makes it one of the most accurate GPS devices available in the market.

c. Spark Nano 4.0 GPS Tracker [14]

It is a new and improved version of most popular GPS tracker and is one of the best way to monitor people, car or assets. It is capable of viewing location on any smart phone, tablet or PC. It has a default one minute update frequency rate and provides instant speed and location alerts via email.

d. BrickHouse HCT Pro plus [15]

It is the most covert GPS tracker which features a 12 hour battery back up and improved accuracy. It is completely invisible when installed. Being hardwires, it never runs out of power. It has an extra antenna which increases its accuracy and precision.

e. SilverCloud Real-time GPS tracker

It is a professional vehicle monitoring system with easy-to-use tracker. Also, it has a built-in fleet management functionality, location sharing and mobile capability. It has a lightning fast 10 seconds location update rate.

So, as discussed above, with the help of GPS tracking system placed in the vehicle (either hardwired or portable), exact location of the vehicle can be tracked via a web-browser or web-enabled smart phone from anywhere in the world, 24/7. These systems also generate essential reports that presents an accurate analysis of fleet operations. It lowers the business costs, alerts traffic jams and accidents and forwards the best alternate routes to minimize delays. It also gives great customer satisfaction by dispatching the vehicles quickly and on time. It increases business competitiveness too. Intensive research is also growing in the area of Route tracking. or traffic estimation.

Cell-based algorithm[6] is used to estimate road traffic condition from multiple Global Positioning System (GPS)-equipped taxis. The GPS data is received intermittently at a central server from approximately 4,000 taxis running within Bangkok metropolitan area. The algorithm divides the Bangkok area into 6,400 square cells. Traffic condition considered here is travel time between two cells. The A* algorithm is used to predict the travel path between any pair of origin-destination. The incremental weighted update algorithm is used to update and estimate travel time between cells. The algorithm can estimate traffic condition of all areas anytime, even if there is no GPS data sent from that area. The result shows that the cell-based algorithm can achieve a good traffic estimation covers all areas and times.

Another traffic congestion identification system, a new model for estimating link average speed [7] is designed considering the shortcomings of traditional models, and a method for identifying traffic congestion is put forward with link average speed obtained above. Then, the method is verified using measured GPS and GIS data based on a part road network of a large urban. The results show that the precision of traffic congestion identification by the method in this paper is much higher.

Also, there is another method which considers the fusion of two types of data for the purpose of realtime traffic fusion [8] and prediction: GPS data that is provided as point speeds, rather than trajectories, as well as (non real-time) traffic data such as is available from fixed sensors.

Through vehicle-mounted GPS date and map-matching technique, vehicle GPS locating point was matched within the corresponding traffic region by using circle split method [9], and the issue of acquisition of region traffic state was resolved. In this paper, technique process of acquisition of region traffic state based on map matching was sketched firstly, then region matching method was deeply researched, circle split method was put forward to perform region matching, and speed distribution curve was obtained by calculation, thus feasibility of circle split method was verified. The Circle split method divides different areas with certain circles, centres and radiuses are determined by the shapes and dimensions of different areas. The division principle is to fully cover a certain traffic area with circles as few as possible.

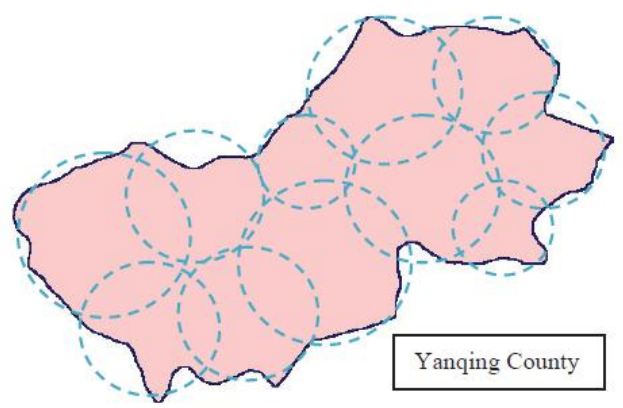

Fig 2.1: Circle split method 
To achieve smart cities, real-world trace data sensed from the GPS-enabled taxi system, which conveys underlying dynamics of people movements, could be used to make urban transportation services smarter. As an example, it will be very helpful for passengers to know how long it will take to find a taxi at a spot, since they can plan their schedule and choose the best spot to wait. In this paper, we present a method to predict the waiting time for a passenger at a given time and spot from historical taxi trajectories. The arrival model of passengers and that of vacant taxis are built from the events that taxis arrive at and leave a spot. With the models, we could simulate the passenger waiting queue for a spot and infer the waiting time. The experiment with a large-scale real taxi GPS trace dataset is carried out to verify the proposed method.

\section{Proposed System}

The proposed system has the following requirements. It uses a Track Port GPS Vehicle which can track the current location of the bus. The data is sent via GPRS to the server using the POST method of the HTTP protocol. A web application will be developed using JavaScript, Ajax, and MySQL. Google Maps will be incorporated in it.

The Block diagram of the working model is as follows:

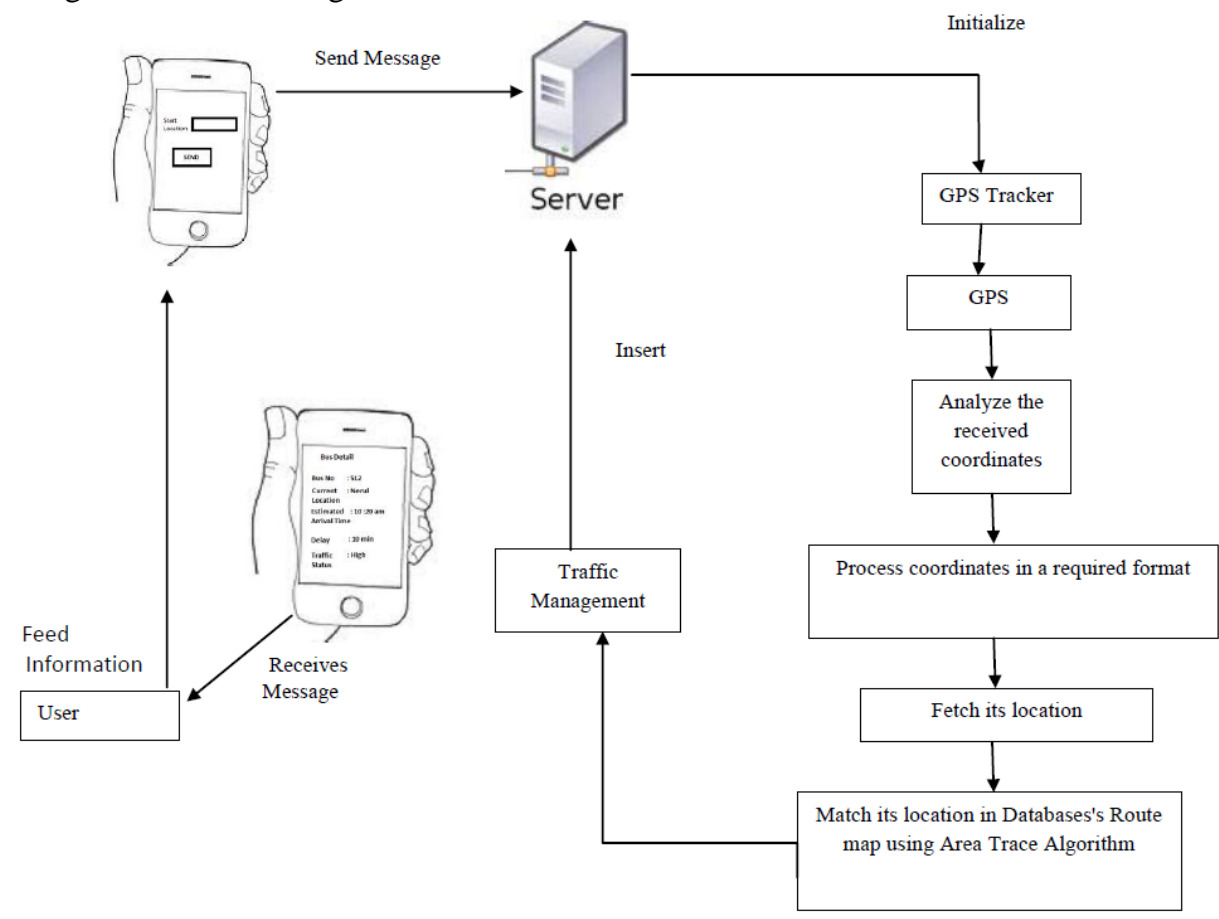

Fig 3.1: Block Diagram for the Bus Tracking System

\section{User}

The user for our proposed system is a common man who has a basic cell phone with just calling and messaging capacity. This is the basic requirement for the user to get the real-time data from the server. This is the advantage of the system that unlike other applications, this system does not requires high-end GPRS enabled smart phone or GPS devices. In a city like Mumbai, traffic has always been a major problem which leads in delays in day to day activities of a common man. As discussed earlier that a majority of the population travels by bus, it is a part of a common man's life. The user over here is the common man who wants to travel from one place to another by bus. Being his regular traveller, he knows the bus number to be cached, however the location of the bus is a major concern for him. Location alone doesn't suffice the requirements. The user must also know traffic conditions so that the expected arrival time of the bus can be estimated. This expected arrival time will help the user in deciding whether the wait time for the bus is worth it or he should think of some other means of transport for travelling.

One more problem which gets highlighted now is the vacancy of the seats in the bus. Although the waiting time for the bus is less, it is of no use if the bus which arrived after an amount of waiting time has no place for passengers to be accommodated, it being already full. The bus has a fixed number of seats and a limited number of standees allowed. Now, for the user to take a smart call for the wait time, he must also be notified with the available vacancy of seats inside the bus. 
So, the user can now be aware of certain parameters like the current location of the bus, the expected arrival time of the bus and the number of available vacant seats in the bus. These parameters will help the user to classify the wait time as worth it or not. What the user has to do to obtain the information of the above mentioned paramaters is to simply send a text message. This text message has to be sent in a specified format to the server as "BUS bus_number". This message contacts the server and request the required data. The server in turn contacts the database and gathers the required data in a format as " BUS bus_number LOC nearest_bus_stop_for_bus_location AV-SEATS max_count STANDEE max_standee_allowed PASS current_count_of_passengers TIME expected_arrival_time", where BUS stands for bus number requested, LOC gives the nearest location where the bus is located, AV-SEATS shows the maximum number of seats available in that bus, STANDEE gives the count of maximum number of passengers who are allowed to travel standing in the bus, PASS gives the current number of passengers travelling in that bus and TIME gives the expected arrival time for the bus. All this not only saves the users time, efforts and money but also increases his flexibility.

\section{GPS Tracker}

Every bus is equipped with a Track Port GPS Vehicle Tracker. The reason for selecting this tracker is that it has a tracking position update rate as fast as 10seconds. It can store unlimited restricted data on a secure server and is accurate within 7 feet of the device. It has a rechargeable Lithium-Ion battery. It works on real time location monitoring and is a low-cost tracker.

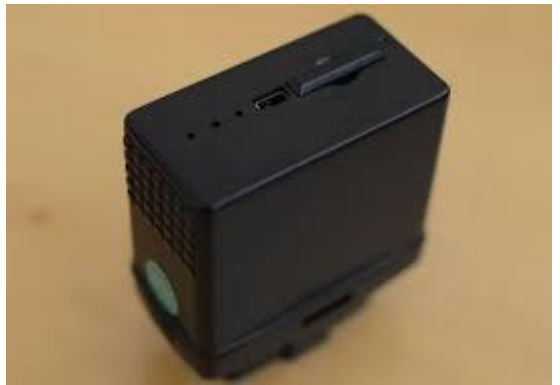

Fig 3.2: Track Port GPS Vehicle Tracker

This GPS tracker sends the server the current GPS coordinates to the server every 10seconds where the further processing on the obtained coordinates are performed.

\section{Sensor}

The sensor module has sensors which are used for scanning as input an user or passenger. There are two sensors fitted at the entry and exit doors of a bus. These sensors have the duty of reading an incoming or outgoing passenger in a bus or out of the bus respectively. Every time a passenger boards a bus, the sensor module increments the count and this process is repeated for every passenger. The sensor module also decrements the count when a passenger leaves the bus.

Technically speaking, the sensor module is connected to the counter which has two basic functions namely, the maximum number of passengers who can be accommodated in a bus and the current count of the passengers who are travelling in the bus. Thus, the current number of passengers can be estimated by the two sensors which has the entry and exit count of the passengers. Also, the total number of available seats can be estimated by the difference between the maximum number of passengers who can be accommodated in a bus and the current number of passengers who are currently travelling in the bus. This information is further saved in the database and is periodically updated every two minutes.

\section{Server}

As shown in the block diagram, the server initially communicates with the user. When an user sends a text message to the server for obtaining information about a specific bus number, the server contacts the database and retrieves the requested data. This data is sent by the serer to the user in a specific format. 


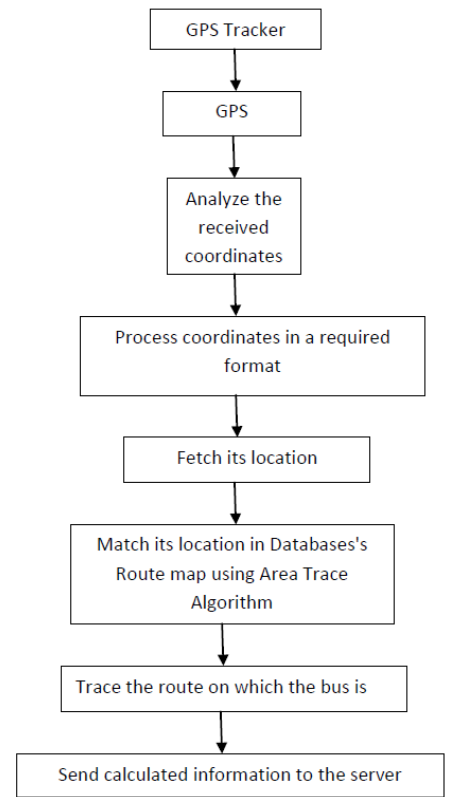

Fig 3.3: Bus route trace using Area Trace Algorithm

The server also communicates with the GPS as shown in the Figure 3.3. The GPS gets the update from the GPS tracker every 10seconds about the current location of the bus. These coordinates are further sent to the server. The server initially analyzes the received coordinates and does processing on them to get them in a required format.

These coordinates are mapped with the locations stored in the database's route map using Area Trace Algorithm and Haversine distance method. Finally, the route on which the bus is currently running is traced and this information is further sent to the server.

\subsection{Area Trace Algorithm}

Area trace algorithm takes as input the Bus Route Map of Mumbai and further divides Route map into a fixed number of areas based on its classification parameters whose coordinates are stored in the database. This helps the server to get down the search from a broader to narrower spectrum where the area to which the current location belongs can be easily traced.

The database also stores the coordinates of each of the bus stop locations and some of the prominent locations in Mumbai which will be helpful for calculating the exact location of the bus using the Haversine Distance method.

The algorithm works as follows:

1. The Bus Route Map of Mumbai is given as input to the Rear Trace Algorithm.

2. Divide the area in the Route Map into fixed number of areas based on some classification paramaters as per the range of the coordinates and a make a set of each area.

3. Obtain the current location coordinates of the bus from the GPS.

4. Compare the current coordinates to the range of the coordinate set stored obtained in the database to get the area set of Mumbai in which the bus is located.

5. Return the area set obtained to the server.

6. Choose the prominent locations and bus stop coordinates in that area set and compare each of these locations to the obtained current location of the bus using Haversine distance method.

7. Calculate the smallest Haversine distance. The bus stop or prominent location from the database which is most near to the current location of the bus will be having the smallest Haversine distance.

8. Return the smallest distance and the name of the location to the server which would be further stored in the database.

\subsection{Haversine Distance Algorithm:}

Haversian distance algorithm is used to calculate the great circle distances between any two points using the 'Haversine' formula [17]. The formula assumes that the earth is a sphere i.e egg-shaped, but proves to be accurate enough for all purposes. Haversine formula takes as input any two GPS coordinates. These coordinates 
can be accepted in a variety of formats either as deg-min-sec suffixed with N/S/E/W (e.g. 40 $44^{\prime} 55^{\prime \prime} \mathrm{N}, 7359$ $11 \mathrm{~W}$ ), or signed decimal degrees without compass direction, where negative indicates west/south (e.g. 40.7486, -73.9864). We use the former one which takes as input the latitude and longitude coordinates.

Lat 1: $530902 \mathrm{~N}$
Lat 2: $521217 \mathrm{~N} \quad$ Long 1: $0015040 \mathrm{~W}$
calculate distance $170.2 \mathrm{~km}$

Fig 3.4: Haversine Distance Calculation

Figure 3.4 makes the working more clear.

Haversine formula works as:

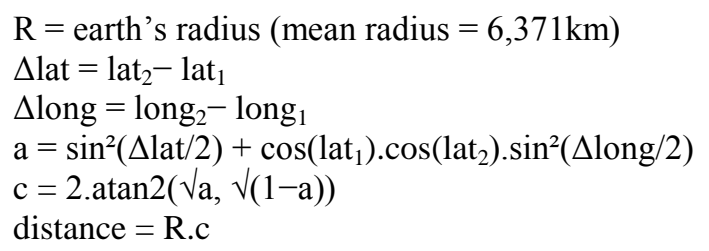

Note that angles need to be in radians to pass to trig functions.

The Haversine formula remains particularly well-conditioned for numerical computation even at small distances.

Further, as per Spherical law of cosines,

distance $=\operatorname{acos}\left(\sin \left(l_{a t}\right) \cdot \sin \left(l_{a t}\right)+\cos \left(l_{a t}\right) \cdot \cos \left(l_{2} t_{2}\right) \cdot \cos \left(\operatorname{long}_{2}-\operatorname{long}_{1}\right)\right) \cdot R$

The algorithm works as follows:

1. Start

2. Get the current location coordinates of the bus from the GPS.

3. Get the coordinates of the prominent locations and bus stop from the area set to which the current location coordinates of the bus correspond.

4. Calculate the Haversian distance between the coordinates obtained from step 1 to each of the coordinates obtained in step 2.

5. Calculate the minimum Haversian distance from the results of step 4 .

6. Return the minimum obtained distance from step 5 to the server.

Lastly, the server also communicates with the database for the periodic updating to be saved onto it. It also interacts with the database to fetch the required information requested by the user. It also interacts with the Traffic Management System for maintaining a track of the traffic conditions.

\section{Traffic Management System}

The Traffic Management System module stores the information of the current traffic condition in the database. This module is also used for estimating the expected arrival time of the bus. The Traffic Management System module divides the traffic conditions into six categories namely No Traffic, Very Low Traffic, Low Traffic, Moderate Traffic, Heavy Traffic and Very Heavy Traffic.

The module works a follows. When a bus is stuck up in a traffic, its location remains unchanged for a longer period of time. This principle is used by the Traffic Management System module to estimate the amount of traffic. For a city like Mumbai, we assume that the decent speed for vehicle to be clubbed under the category of No Traffic zone is $50 \mathrm{~km} / \mathrm{hr}$. As per the Traffic Authority rules of Mumbai, a traffic signals wait duration is anything between 30 seconds to 120 seconds, 120 seconds being the maximum waiting time. So, it can be now stated that if a bus is not moving from one location for more than 120 seconds, it is bound to be stuck up in traffic. Now the question arises as to in how much traffic is the bus stuck up? For this, the records of the current location coordinates of the bus are sent every 10 seconds in the database.

When a vehicle is running at the speed of $50 \mathrm{~km} / \mathrm{hr}$, the time it takes to cover 100meters distance is 7.1 seconds. similarly, when a vehicle is running at the speed of $40 \mathrm{~km} / \mathrm{hr}, 30 \mathrm{~km} / \mathrm{hr}, 20 \mathrm{~km} / \mathrm{hr}, 10 \mathrm{~km} / \mathrm{hr}$ and $5 \mathrm{~km} / \mathrm{hr}$; it covers 100 meters distance in $9,12,17.98,35.97$ and 72 seconds respectively. These results are simply obtained from the simple speed and distance formula. Based on the above calculations, it can be said that a vehicle covering a distance of 100 meters in $7.1,9,12,17.98,35.97$ and 72 seconds can be classified to be in No Traffic category, Very Low Traffic category, Low Traffic category, Moderate Traffic category, Heavy Traffic category and Very Heavy Traffic category respectively.

The GPS Tracker sends the current location of the bus every 10 seconds to the server, The server processes this data and stores it in the database. The Traffic Management System works on this data which is stored in the database. It takes an input the past 120 seconds records from the database and predicts the traffic 
conditions based on the distance travelled by the bus. So, it basically works on the samples of the various coordinate locations stored in the database. Assume the following path flow to understand the working of the Traffic Management System module:

A-->B-->C-->D------------------->E.

Here, A, B, C and D are the location coordinates of the bus at an interval of every 120 seconds. E represents the coordinates of the user who wants to know the expected arrival time of the bus. The Haversine distance between $\mathrm{A}$ to $\mathrm{B}, \mathrm{B}$ to $\mathrm{C}$ and $\mathrm{C}$ to $\mathrm{D}$ is calculated. This gives the actual distance travelled in meters from A to $\mathrm{B}, \mathrm{B}$ to $\mathrm{C}$, and $\mathrm{C}$ to $\mathrm{D}$. Also, the information about the time required to travel each of this distance is already stored in the database. These all values of distances and time will be speed to calculate the cumulative speed of the bus to travel from A to D. This obtained information is further used for classifying the bus in No Traffic to Very Heavy Traffic category. Knowing the location coordinates for D and E, we travel the Haversine distance between D and E. Also, we have a record of cumulative speed of the bus. Again, using the standard speed and distance formula, the time taken by the bus to travel from $\mathrm{D}$ to $\mathrm{E}$ can be predicted. This time is nothing but the expected arrival time for the bus. This time is further stored in the database and sent to the user when a request is to be processed.

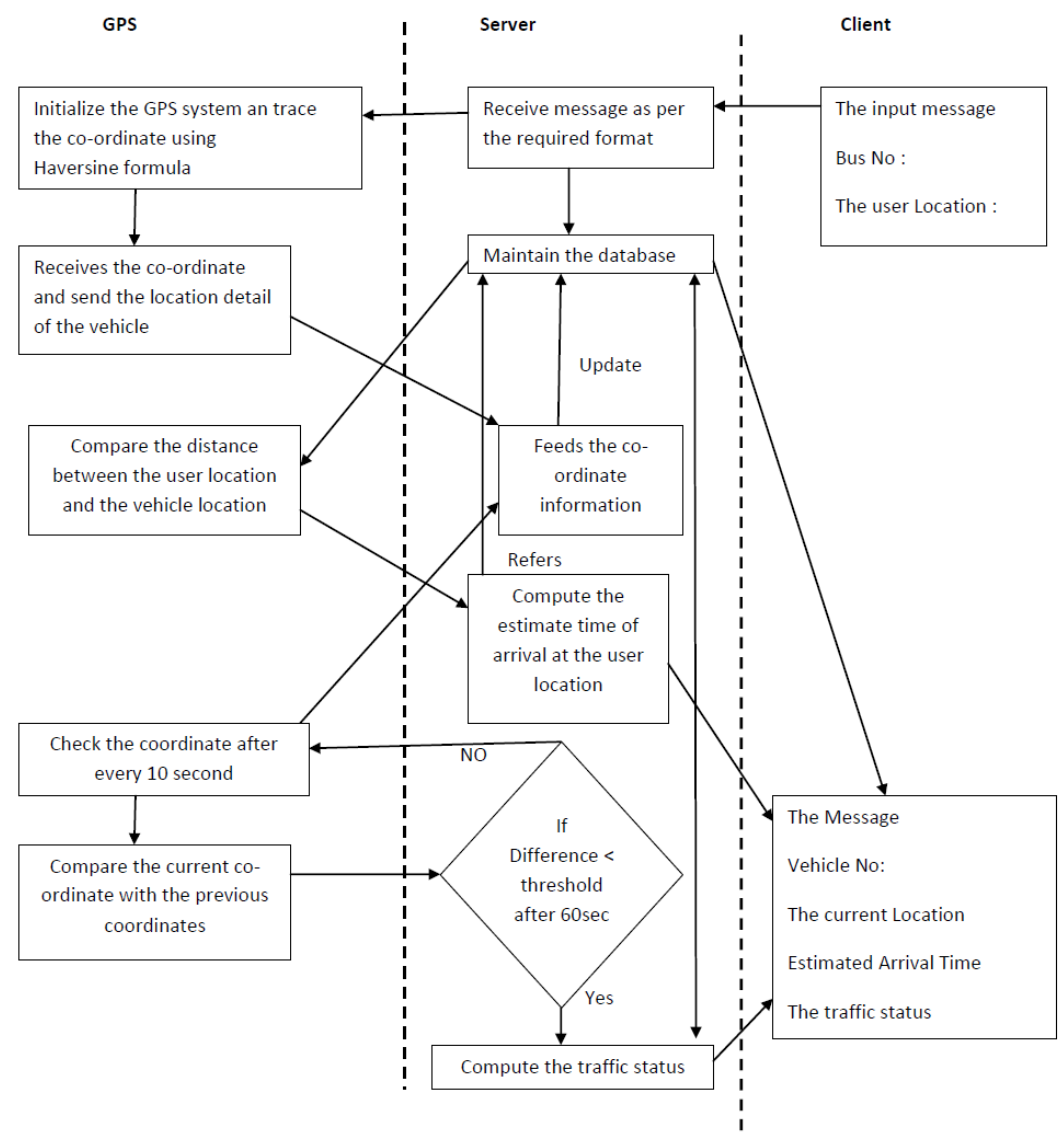

Fig 3.5: Activity Diagram for the Overall System

In Fig 3.5, the proposed system is divided into three major part i.e. Client, Server and GPS . The working starts with the user using any mobile phone(thus platform independent) with or without internet connection, thus opens the application. The application asks user to feed the basic information i.e. Bus No. and the user's location. The moment this information is feed then submit this information to the server using internet or the ISP services of the user. Since the system is using both the mode of transferring message its not important that every mobile should have the internet services activated.

At the server, the information which is triggered to server by the user gets the updated information which is stored in the database of the server. This database is updated after every $10 \mathrm{sec}$, the information like the bus location, the distance to be travelled by the bus, the speed, the estimated arrival time of each bus at the respective bus stop, the traffic information etc. The main evaluation parameter in this section is the estimation of arrival time of the bus with respect to user location which is performed by the area trace algorithm. The another major section is the traffic management, which is using the co-ordinate of the the bus and keeps on comparing with the previous information if the co-ordinate have a significant difference indicate the motion of the bus. Thus if the difference is minimum indicate that may be the bus is in static position or is stuck in a traffic. If the bus has stop in a particular bus stop for a period of say $60 \mathrm{sec}$ indicate that the bus is stop but if the difference is 
minimum for a a substantial period of time indicate either the bus is not working or the traffic status is very high thus result in delay. This delay information is stored in the database of the server so that the user is aware of the live condition and can make judgment accordingly. The server thus sends all the detail such as the bus no, the current location of the bus, the estimated arrival time at the user location, the delay, the traffic status to the user.

The role of the last section the GPS i.e. the Global Positioning System is to trace the bus and get the coordinate of the bus and store the data in the server for every $10 \mathrm{sec}$. Thus updating the information in the run time making the system more accurate and easy. This entire system will ease the user to plan their travel better in an efficient manner giving them all the detail within a click of a button.

\section{Conclusion}

In today's world where every second counts, it's important that time is managed properly. This system will help user to exactly plan there their trip in better and a cheaper way. The system will also help user to estimate their schedule according to their requirement in efficient manner. For further improvements one can user the sensor information to get the information of the capacity of the bus so that user is aware of whether the bus they are waiting for is empty or is crowded so that they can plan accordingly about their alternate option.

\section{References:}

[1] Raj Kishen Moloo, Varun Kumar Digumber, Low-Cost Mobile GPS Tracking Solution, International Conference on Business Computing and Global Informatization, 2011, 516-519.

[2] Prathusha Perugu, An Innovative Method using GPS Tracking,WINS Technologies for Border Security and Tracking of Vehicles, IEEE, 2010, 130-133.

[3] Katina Michael, Andrew McNamee, MG Michael, The Emerging Ethics of Humancentric GPS Tracking and Monitoring, Proceedings of the International Conference on Mobile Business (ICMB'06), 2006.

[4] Hassan A. Karimi, J. Tom Lockhart, GPS-Based Tracking1 Systems for Taxi Cab Fleet Operations, IEEE - IEE Vehicle Navigation \& Information Systems Conference, Ottawa - VNIS ‘93, 1993.

[5] Pham Hoang Oat, Micheal Drieberg and Nguyen Chi Cuong, Development of Vehicle Tracking System using GPS and GSM Modem, IEEE Conference on Open Systems (ICOS), 2013, 89-94.

[6] S. Sananmongkhonchai, P. Tangamchit, P. Pongpaibool, Cell-based Traffic Estimation from Multiple GPS-Equipped Cars, IEEE, 2009, 1-6.

[7] CHANG Ande2, JIANG Guiyan1, 2, NIU Shifeng2, Traffic Congestion Identification Method Based on GPS Equipped Floating Car, International Conference on Intelligent Computation Technology and Automation, 2010, 1069-1071.

[8] Wei Shen, Laura Wynter, Real-Time Road Traffic Fusion and Prediction with GPS and Fixed-Sensor Data, IEEE, 1468-1475.

[9] Hu Yue, Qin Yong, Lv Yuqiang, Dong Honghui, Xu Jie, Regional Traffic State Estimation With A GPS-based Method, IEEE, 2012, 3774-3777.

[10] Guande Qi, Gang Pan*, Shijian Li, Zhaohui Wu, Daqing Zhang, Lin Sun, Laurence Tianruo Yang, How Long a Passenger Waits for a Vacant Taxi?, IEEE International Conference on Green Computing and Communications and IEEE Internet of Things and IEEE Cyber, Physical and Social Computing, 2013, 1029-1036.

[11] Ikuko Shigihara, Akiko Arai, Osamu Saitou, Yuji Kuwahara and Masaru Kamada, A Dynamic Bus Guide Based on Real-Time Bus Locations, 16th International Conference on Network-Based Information Systems, 2013, 436-438.

[12] http://www.brickhousesecurity.com/product/trackport+gps+tracker.do?sortby=bestSellers\&from=fn

[13] http://www.brickhousesecurity.com/product/brickhouse+hct+plus.do?sortby=bestSellers\&from=fn

[14] http://www.brickhousesecurity.com/product/spark+nano+gps+tracking+device.do?sortby=bestSellers\&refType=1

[15] http://www.brickhousesecurity.com/product/brickhouse+hct+pro+plus.do?sortby=bestSellers\&refType=1

[16] http://www.brickhousesecurity.com/category/gps+tracking/vehicle+tracking+devices.do

[17] http://www.ig.utexas.edu/outreach/googleearth/latlong.html 